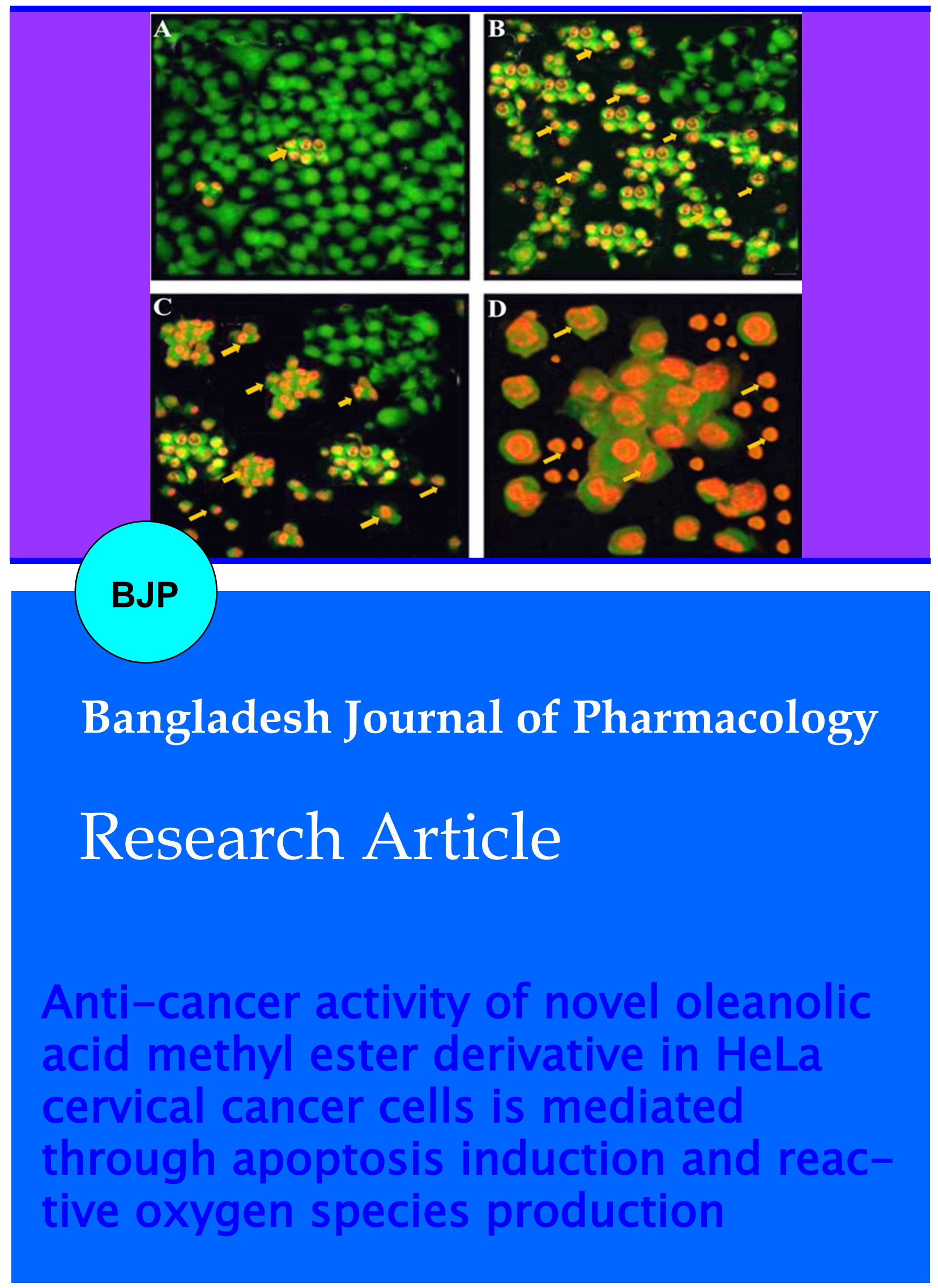




\title{
Anti-cancer activity of novel oleanolic acid methyl ester derivative in HeLa cervical cancer cells is mediated through apoptosis induction and reactive oxygen species production
}

\author{
Xin Song1, Chang-Chun Liu ${ }^{2}$, Yu-Rong Hong3 and Xiao-Cong Zhu ${ }^{4}$ \\ Department of ${ }^{1}$ Special Medical Treatment, ${ }^{2}$ Neurology, and ${ }^{3}$ Renal Medicine, Coal General Hospital, \\ Beijing 100 028, China; ${ }^{4}$ Department of Uropoiesis Surgical, Jishuitan Hospital, Beijing 100 850, China.
}

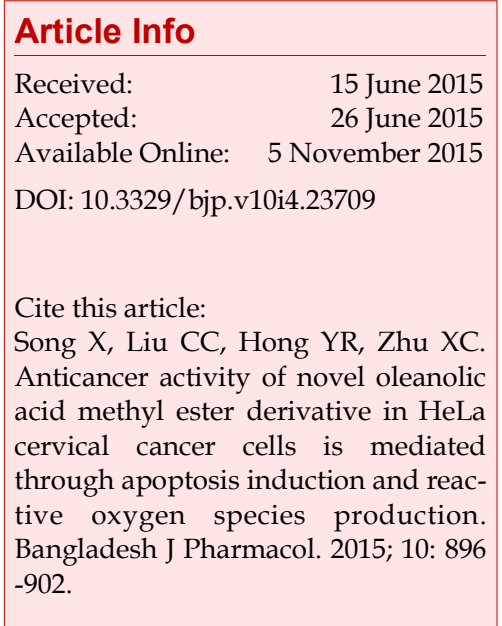

\begin{abstract}
The objective of this study was to determine the anticancer and apoptotic effects of a novel oleanolic acid methyl vanillate derivative in human cervical cancer cells (HeLa). We also evaluated effect of the compound on reactive oxygen species (ROS) generation. MTT and LDH assay were used to evaluate the effect of this compound on HeLa cancer cell cytotoxicity. Confocal microscopy and flow cytometry evaluated the effect on apoptosis induction, while as ROS production was detected by using fluorescent CM-DCFH2-DA. The compound induced potent cytotoxic effects in HeLa cells in a dosedependent as well as time-dependent manner. The compound also induced characteristic morphological changes indicative of apoptosis. The compound induced both early and late apoptosis in a concentration-dependent and timedependent manner. The compound led to a significant and concentration dependent increase in the ROS production as the concentration of oleanolic acid methyl vanillate derivative was increased from 0 to 15,60 and $120 \mu \mathrm{M}$.
\end{abstract}

\section{Introduction}

Cervical cancer is believed to be the second most common cancer found in women all across the globe. This cancer mostly targets the poor and developing countries and around 75 to $80 \%$ cases are found in these poor countries. Cervical cancer affects relatively young women and as such is an important cause of early death (World Health Organization, 2006). Human papillomavirus (HPV) infection has been found to have a distinct link with cervical cancer (Doorbar, 2006). The initial symptoms of cervical cancer are not so evident, however, later symptoms include pelvic pain, abnormal vaginal bleeding, etc. During the progression of cervical cancer, a chain of anomalous events are induced, including disruption of cell cycle, disturbance of antitumor immune response, alteration of gene expression, and deregulation of microRNA expression. Despite the fact that surgery and chemo-radiotherapy can cure $80 \%-$
$90 \%$ of women with early stage cervical carcinoma, the recurrent and metastatic malignancy remain a key cause of cancer deaths all across the globe. Imiquimod and gemcitabine which are the immunomodulatory agents have been used for cervical cancer treatment in recent times (Daayana et al., 2010; Suzuki et al., 2007).

Oleanolic acid (3 $\beta$-hydroxy-olean-12-en-28-oic acid) belongs to oleanane triterpene group of natural products mostly abundant in plant kingdom (Somova et al., 2003). Literature survey revealed that oleanolic acid possesses a range of promising biological and medicinal effects including the antiproliferative activity. The molecule has been reported to suppress tumor development in vivo and in mouse skin (Ohigashi et al., 1986; Tokuda et al., 1986; Huang et al., 2006). Oleanolic acid has been shown to exert cytotoxic activities in vivo against three human cancer cell lines namely, nasopharyngeal carcinoma, oral epidermoid carcinoma and 
colorectal carcinoma cells (Chiang et al., 2005). Although oleanolic acid has been reported to exhibit anticancer activity against many cancer cell lines, but its methyl ester derivative has not been worked out so far. Therefore, the objective of the current investigation was to evaluate the anticancer and apoptotic effects of oleanolic acid methyl vanillate ester (OAMVE) in HeLa cervical cancer cells.

\section{Materials and Methods \\ Chemicals and other reagents}

Oleanolic acid was purchased from Chengdu Preferred Biotech Co. Ltd (China). N-acetyl-L-cysteine and tocopherol were purchased from Beyotime Inc, (China). 5(6)-carboxy-2', 7'-dichlorodi-hydrofluorescein diacetate, acridine orange, trypsin, dimethyl sulfoxide (DMSO) were purchased from Sigma-Aldrich (USA). 3-[4, 5dimeth-yl-2-thiazolyl]-2, 5-diphenyl tetrazolium bromide (MTT), was purchased from Molecular Probes (USA). Dulbecco's modified Eagle's medium, fetal bovine serum, penicillin-streptomycin were obtained from Hangzhou Sijiqing Biological Products Co. Ltd, China. All other chemicals and solvents used were of the highest purity grade.

\section{Chemical synthesis of oleanolic acid methyl vanillate} ester

Oleanolic acid methyl ester reacted with methyl chloride in the presence of $\mathrm{K}_{2} \mathrm{CO}_{3}$ and acetone and was continuously stirred at room temperature for 15 hours to protect the hydroxyl groups of the oleanolic acid methyl ester as methoxy methyl chloride. After that the ester group was subject to hydrolysis with $\mathrm{C}_{2} \mathrm{H}_{5} \mathrm{OH} /$ aq. $\mathrm{KOH}$ for another 10 hours and then acidified to give oleanolic acid with its -OH group protected as methoxy methyl ether. This derivative was further treated with the -OH groups of methyl vanillate in the presence of DMAP (dimethyl aminopyridine), N, N-dicyclohexylcarbodiimide (DCC) and pyridine in dichloromethane at room temperature for 3 hours. The yellowish white compound thus obtained was finally deprotected using methanol and $5 \% \mathrm{HCl}$ to yield the final product (dark yellow solid) (Scheme 1).

\section{Cell line and culture conditions}

Human cervical cancer cell line (HeLa) was purchased from the Shanghai Institute of Cell Resource Center of Life Science (Shanghai, China), and was kept at $37^{\circ} \mathrm{C}$ in a humidified atmosphere containing $5 \% \mathrm{CO}_{2}$. The cells were cultured in Dulbecco's modified Eagle's medium (Sigma-Aldrich, USA) supplemented with 5\% fetal bovine serum, $2 \%$ penicillin/streptomycin and $2 \%$ amphotericin B.

\section{Cell viability testing by MTT assay}

The inhibitory effects of oleanolic acid methyl vanillate ester on HeLa cell viability were examined using MTT assay. Cells $\left(1 \times 10^{6}\right.$ cells/well $)$ were seeded into 96well plates for 24 hours before treatment. After treatment with various concentrations of oleanolic acid methyl ester $(0,5,15,30,60,90$ and $120 \mu \mathrm{M})$ for 24 and 48 hours, the cell plates were treated with MTT solution ( $5 \mu \mathrm{L} ; 5 \mathrm{mg} / \mathrm{mL}$ in phosphate-buffered saline solution) for an additional 3 hours at $37^{\circ} \mathrm{C}$. The formazan crystals in viable cells were solubilized with DMSO $(150 \mu \mathrm{L})$ and the absorbance was measured on a microplate reader (ELX 800; Bio-tek Instruments, Inc., Winooski, VT, USA) at a wavelength of $490 \mathrm{~nm}$. The effects of oleanolic acid methyl ester on cell viability were calculated using the following equation $\left(\mathrm{OD}_{490}\right.$, optical density at $490 \mathrm{~nm}$ ):

\section{LDH release assay}

HeLa cells were seeded into 96-well plate and subjected to treatment with oleanolic acid methyl ester for 24 hours, then $\mathrm{LDH}$ release $(10 \mu \mathrm{L})$ reagent was added in each well. The cell culture plates were then centrifuged after 1 hour at $800 \mathrm{xg}$ for $15 \mathrm{~min}$ and $150 \mu \mathrm{L}$ of the supernatant from each well was taken out and put into a new black 96-well plate. Afterwards, $50 \mu \mathrm{L}$ of $\mathrm{LDH}$ assay mixture was added to each well and the plate was incubated at $37^{\circ} \mathrm{C}$ for $30 \mathrm{~min}$. The absorbance was measured spectrophotometrically at $490 \mathrm{~nm}$ wavelength.

\section{Measurement of ROS generation}

Fluorescent CM-DCFH2-DA was used to measure intracellular ROS generation in HeLa cancer cells. The cells were seeded in 6-well plates and after cell adhesion, the HeLa cells were pretreated with $20 \mu \mathrm{M}$ CM-DCFH2-DA for 15 min followed by co-incubation with numerous concentrations of oleanolic acid methyl ester for another 1 hour and washed with phosphatebuffered saline three times. The cells were collected and analyzed using a flow cytometry (Becton-Dickinson FACS Calibur flow cytometry (Franklin Lakes, NJ, USA) with wavelength of excitation and emission at 488 $\mathrm{nm}$ and $525 \mathrm{~nm}$ respectively.

\section{Cellular morphological evaluation using confocal fluorescence microscopy}

HeLa cervical cancer cells were seeded on a chamber slide (Thermo Scientific Nunc Lab Tek II) at cell density of $3 \times 10^{3}$ cells per chamber. Acridine orange $(10 \mu \mathrm{g} / \mathrm{mL})$ and propidium iodide $(10 \mu \mathrm{g} / \mathrm{mL})$ were added to each chamber. Then the cells were examined using scanning confocal microscope (Olympus IX 81 microscope).

\section{Annexin V-FITC assay for apoptosis quantification}

Apoptosis induced by oleanolic acid methyl ester in HeLa cervical cancer cells was evaluated using an annexin V-FITC apoptosis detection kit. HeLa cells treated with or without oleanolic acid methyl ester $(0$, 15,60 and $120 \mu \mathrm{M}$ ) were stained with propidium iodide and annexin V-FITC, in accordance with the manufac- 
turer's guidelines. Subsequent to staining the cells, flow cytometry was performed using a Becton-Dickinson FACS Calibur flow cytometry (Franklin Lakes, NJ, USA), and percentages of viable, apoptotic and necrotic cells were analyzed by Cell Quest software.

\section{Statistical analysis}

Data are presented as the mean \pm SEM. All experiments were repeated at least three times. The differences between groups were analyzed by one-way ANOVA, significance of difference was indicated as ${ }^{*} \mathrm{p}<0.05$, ${ }^{* *} \mathrm{p}<0.01$.

\section{Results}

Oleanolic acid methyl vanillate ester induced potent cytotoxic effects in HeLa cervical cancer cells
Chemical synthesis and the molecular structure of oleanolic acid methyl vanillate ester are shown in Scheme 1. MTT assay was performed to evaluate the anticancer effect of oleanolic acid methyl ester in HeLa cervical cancer cells. The results of the MTT assay are shown in Figure 1, which indicate that the compound is a potent cytotoxic agent which inhibits cervical cancer cell proliferation in a concentration dependent as well as time dependent manner. In order to evaluate the efficacy of this compound, $\mathrm{IC}_{50}$ values at different time intervals were calculated and were found to be $56.4 \mu \mathrm{M}$ and $33.8 \mu \mathrm{M}$ at 24 hours and 48 hours time intervals respectively. Further, cytotoxic effect of this compound in HeLa cells were estimated using lactate dehydrogenase release assay. The results which are shown in Figure 2 indicate that oleanolic acid methyl ester led to a concentration dependent release of lactate dehydrogenase into the culture medium.

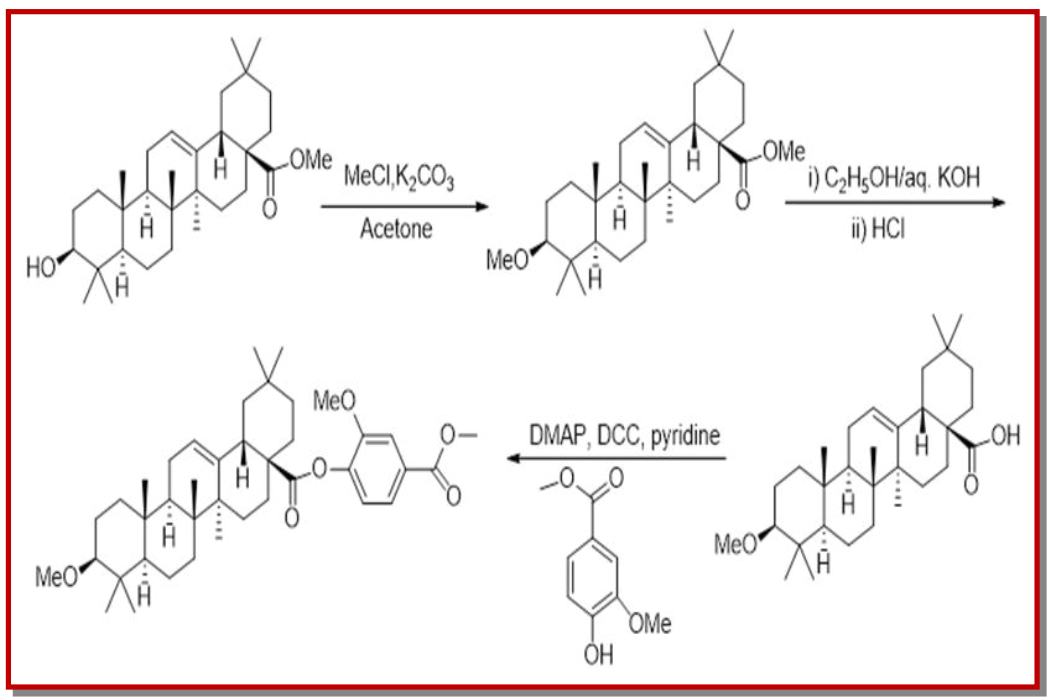

Scheme 1: Chemical synthesis of methyl ester vanillate derivative of oleanolic acid

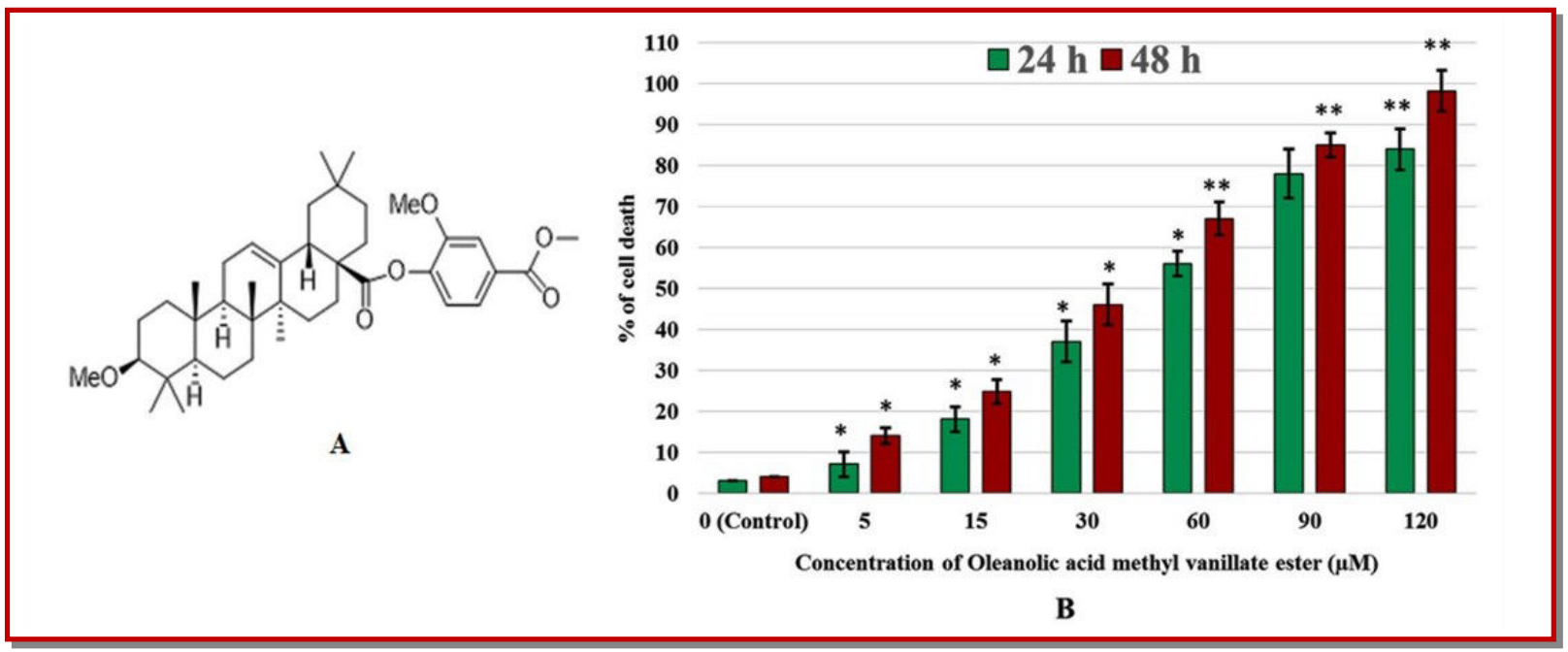

Figure 1: (A) Chemical structure of oleanolic acid methyl vanillate ester (OAMVE); (B) Cytotoxic effect of OAMVE in human cervical cancer cells (HeLa); Data are shown as the mean \pm SD of three independent experiments; ${ }^{*} \mathrm{p}<0.05 ;{ }^{* *} \mathrm{p}<0.01, \mathrm{vs} 0 \mu \mathrm{M}$ (control) 


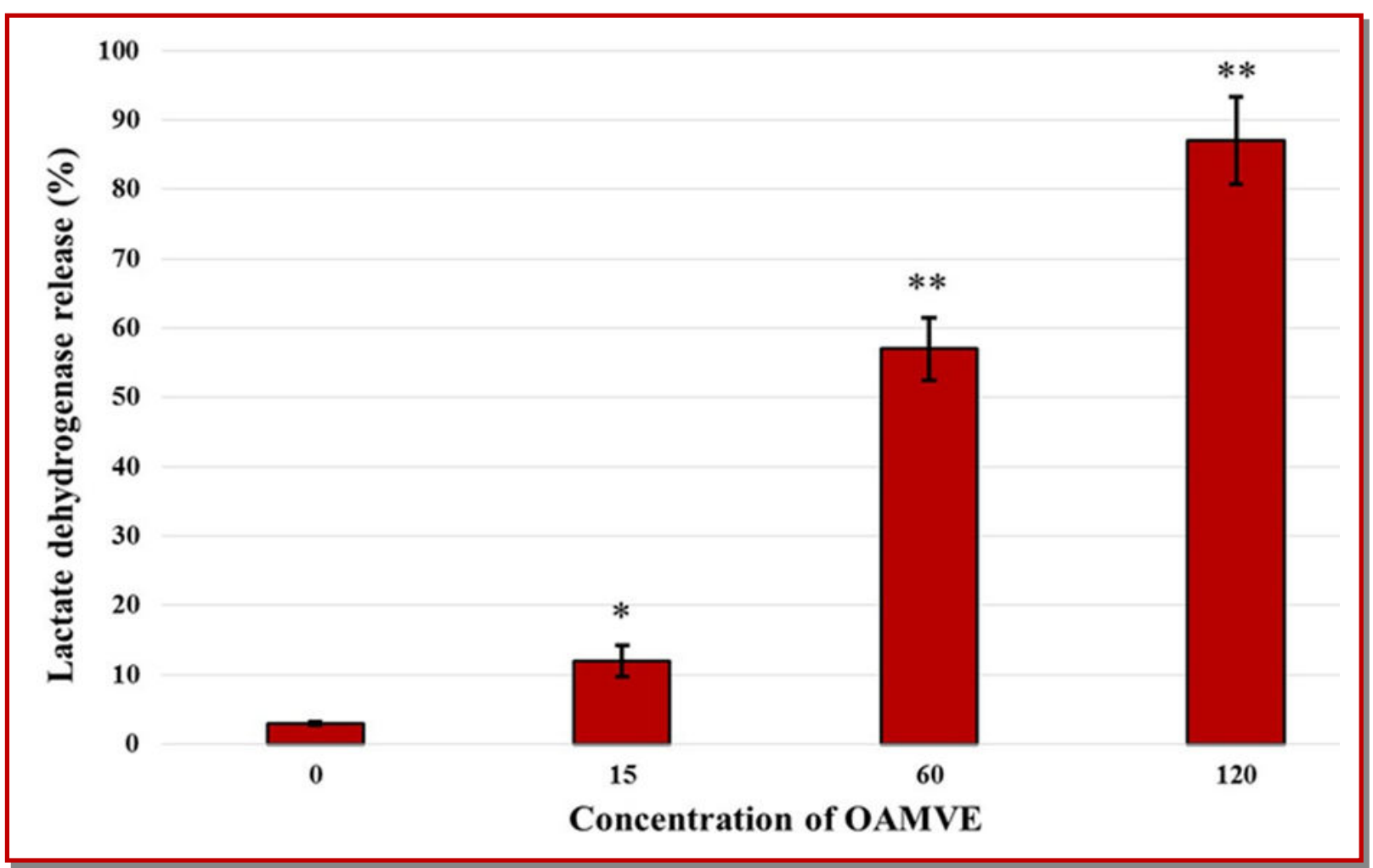

Figure 2: LDH release assay; HeLa cells were treated with various concentrations of OAMVE for 24 hours, LDH release was detected by LDH assay kit; Each point is the mean \pm SEM of three experiments $\left({ }^{*} \mathrm{p}<0.05\right.$; ${ }^{* *} \mathrm{p}<0.01 \mathrm{v} . \mathrm{s}$ control group)

Oleanolic acid methyl ester induced ROS formation in HeL a cervical cancer cells

Intracellular ROS production following treatment with different doses of oleanolic acid methyl ester was detected and calculated by flow cytometry using fluorescent probe CM-DCFH2-DA. As can be seen in Figure 3, oleanolic acid methyl ester led to a significant and concentration-dependent increase in the ROS production as the concentration of the compound was increased from 0 , to 15,60 and $120 \mu \mathrm{M}$. A 3-fold increase in ROS production was observed as the dose of oleanolic acid methyl ester was increased from 60 to 120 $\mu \mathrm{M}$.

Confocal fluorescence microscopic evaluation of the apoptotic cell death induced by oleanolic acid methyl ester

Morphological changes in HeLa cervical cancer cells were detected by confocal fluorescence microscopy stained with acridine orange and propidium iodide. Acridine orange passes through the plasma membrane of living cells and cells that experience apoptosis and then stain cellular DNA. Our results showed that untreated cells (Figure 4A) showed strong green fluorescence while oleanolic acid methyl ester-treated cells showed red fluorescence which increased with increasing dose of oleanolic acid methyl ester (Figure 4B-D). More dead cells were stained with propidium iodide after 48 hours of oleanolic acid methyl ester treatment emitting red fluorescence.

Oleanolic acid methyl ester-induced early and late apoptosis detection evaluation by annexin V-FITC assay

In this study, annexin $\mathrm{V} /$ propidium iodide double staining is utilized to detect early and late stages of apoptosis. Annexin V positive cells represent cells at early stage of apoptosis while cells that are both annexin $\mathrm{V}$ and propidium iodide positive represent cells at late stage of apoptosis or secondary necrosis. Figure 5 shows detection of early and late apoptosis by annexin $\mathrm{V} /$ propidium iodide assay induced by oleanolic acid methyl vanillate ester in HeLa cervical cancer cells. Oleanolic acid methyl ester induced both early and late apoptosis at each time point in a concentration dependent manner. However, at 24 hours time interval, the apoptosis induction was not significant. At 48 and 72 hours of treatment, oleanolic acid methyl ester induced both early and late apoptosis (Figure 5A). Similarly, at higher doses, both early and late apoptosis were observed (Figure 5B).

\section{Discussion}

Cancer involves disorders in the normal process of cell division, which are in turn controlled by DNA of the 


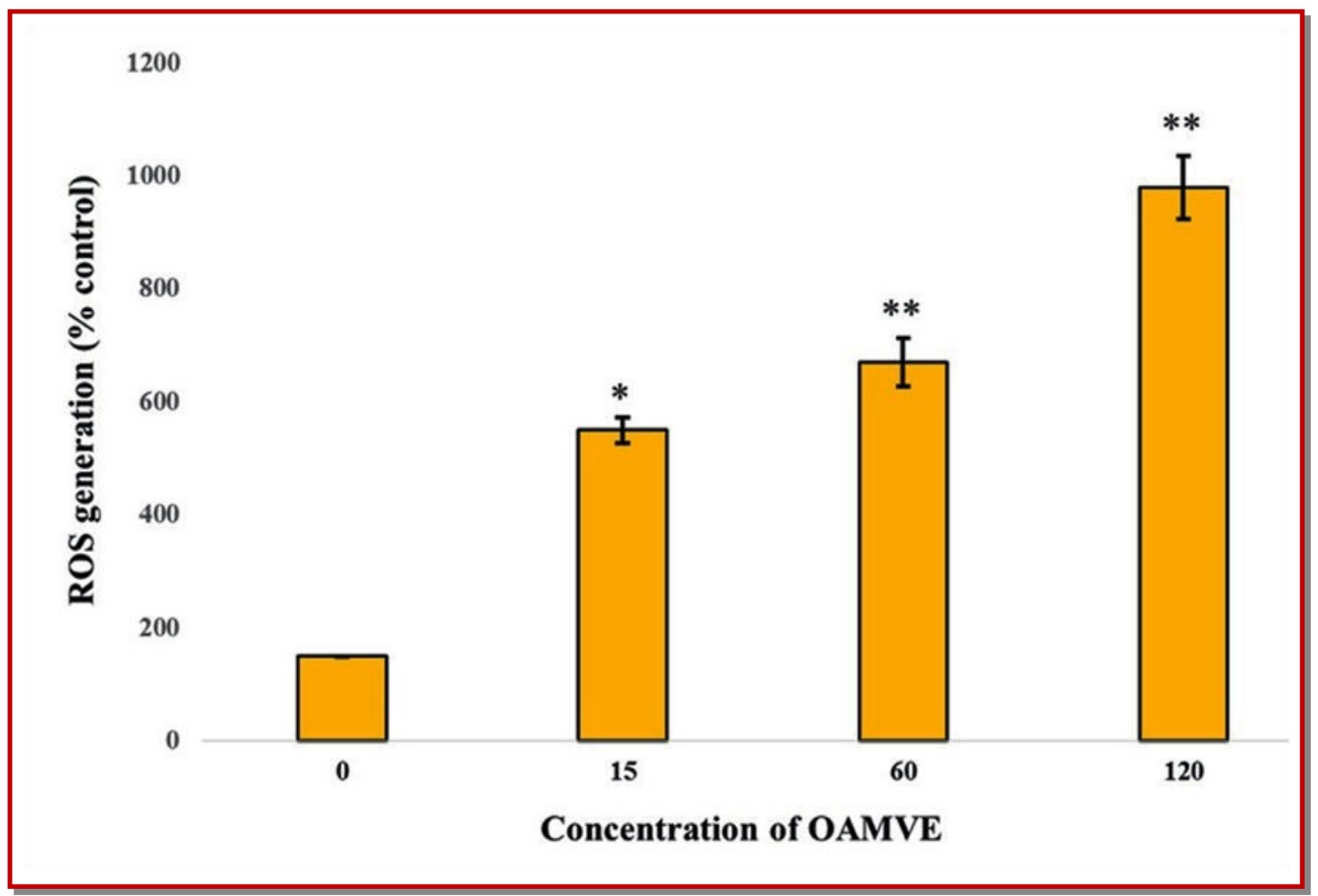

Figure 3: Oleanolic acid methyl vanillate ester (OAMVE) induced ROS generation in HeLa cervical cancer cells. HeLa cells were treated with OAMVE $(0,15,60$ and $120 \mu \mathrm{M})$ for 2 hours, and the ROS generation was estimated using CM-DCFH2-DA staining by flow cytometry. ${ }^{* *} \mathrm{p}<0.01$ vs. control group
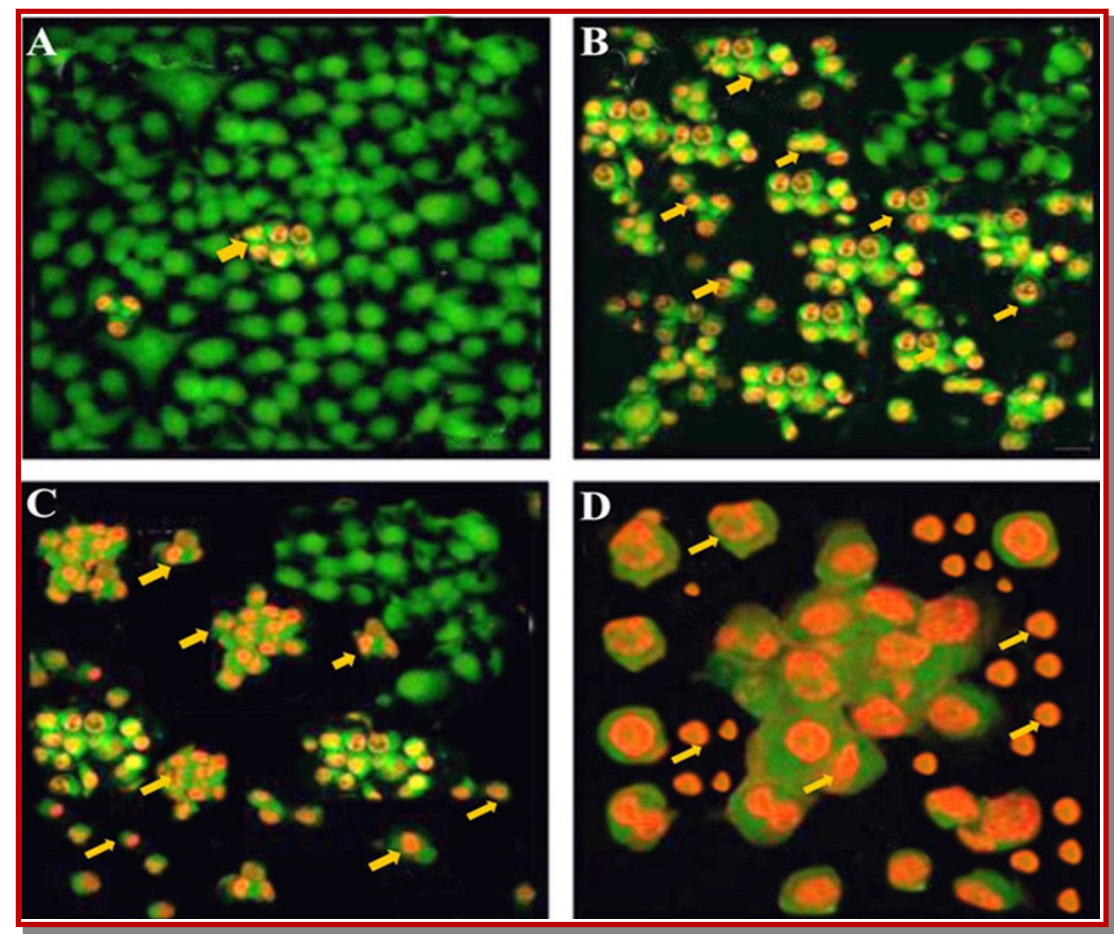

Figure 4: Apoptotic cell death induced by OAMVE in HeLa cervical cancer cells was evaluated by confocal fluorescence microscopy. The cells were stained with AO and PI and observed using confocal microscope (x 400). HeLa cells were treated without (A) and with $15 \mu \mathrm{M}$ (B), $60 \mu \mathrm{M}$ (C) and $120 \mu \mathrm{M}$ (D) of OAMVE for 48 hours; Yellow arrows represent apoptotic cells 


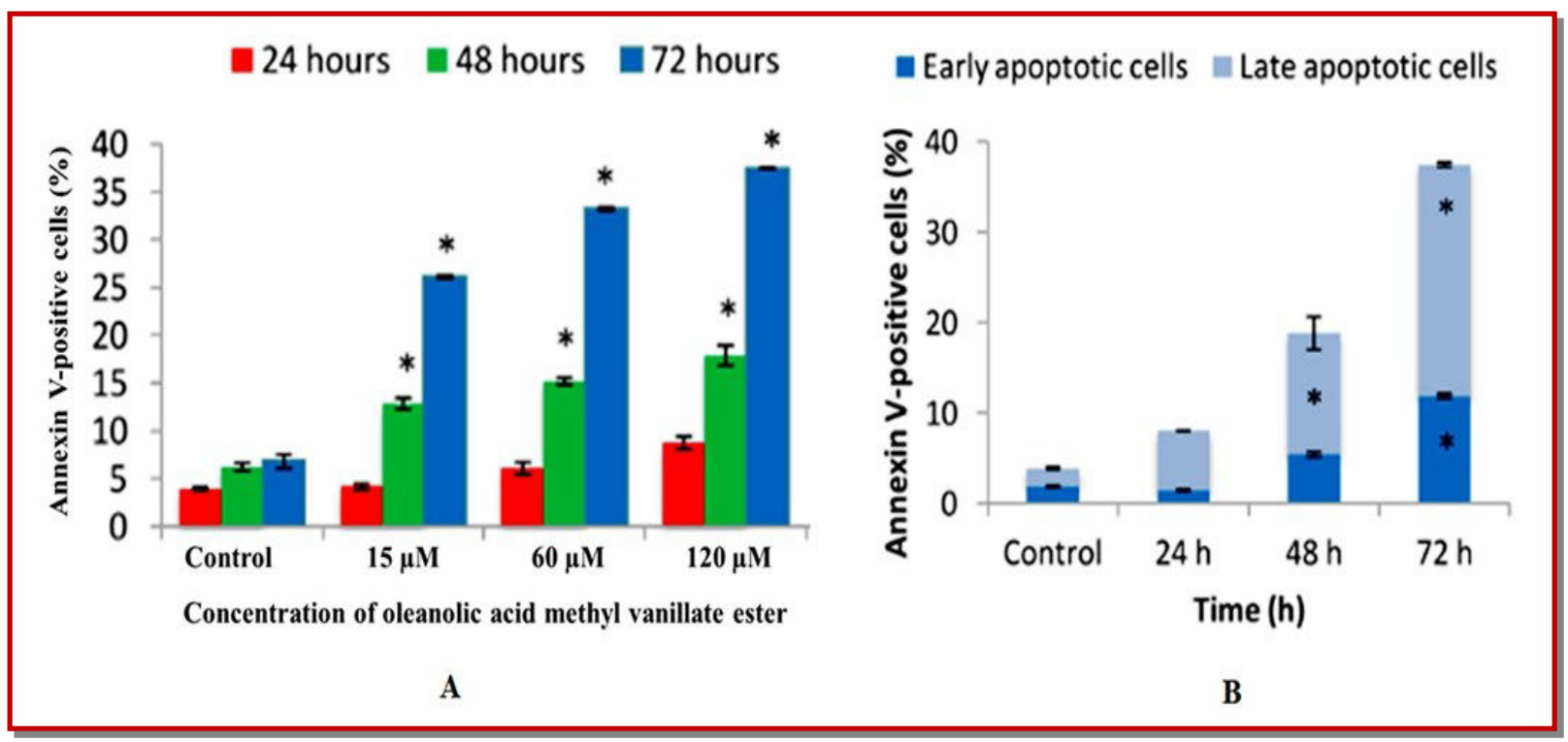

Figure 5: Annexin V/PI assay of oleanolic acid methyl ester-treated HeLa cell line by flow cytometry. After treatment for 24,48 and 72 hours, cells were double stained with annexin V/PI for apoptotic analysis. (A) Induction of apoptosis by oleanolic acid methyl ester in a concentration-dependent manner at 24, 48 and 72 hours of treatment. (B) Induction of early and late apoptosis by oleanolic acid methyl ester at $60 \mu \mathrm{M}$ in a time-dependent manner. Results are represented as mean \pm SD

cell. During the past few decades, cancer research has contributed towards our understanding on cancer biology and cancer genetics. One of the most important factor in the cancer initiation and progression is the apoptosis process and the genes that govern it. Faulty apoptosis indicates a key contributing factor in the development and progression of cancer (Reddy et al., 2003; Lowe and Lin, 2000; Kasibhatla and Tseng, 2003). A hallmark of cancer is that the cancer cells resist towards apoptosis which ultimately results in ineffectiveness of the anticancer drugs to damage tumor cells (Wong and Abdul, 2012). Apoptosis or more frequently known as programmed cell death, functions in tissue development and homeostasis. Several recent scientific studies have advocated the use and manipulation of apoptosis process to target deadly cancer cells. In this direction, many phytochemical compounds have been reported to induce apoptosis in a variety of cancer cells and many of these compounds have even been used in cancer chemoprevention and treatment (Pezzuto, 1997; Prakash et al., 2003).

Our study aimed to investigate the anticancer and apoptotic effects of oleanolic acid methyl vanillate ester derivative against HeLa cervical cancer cells. The MTT and lactate dehydrogenase results showed that oleanolic acid methyl vanillate ester is a potent cytotoxic agent. Fluorescence microscopy revealed that oleanolic acid methyl vanillate ester induces apoptotic cellular morphological alterations in HeLa cells in a dosedependent manner. Oleanolic acid methyl vanillate ester led to a substantial and concentration dependent rise in the ROS production as the concentration of the compound was increased from 0 , to 15,60 and $120 \mu \mathrm{M}$.
Oleanolic acid methyl vanillate ester induced both early and late apoptosis at each time point in a concentration dependent manner as well as in a time-dependent manner at a given dose.

\section{Conclusion}

Our findings reveal that oleanolic acid methyl vanillate ester exhibits anticancer activity in HeLa cervical cancer cells by inducing both early and late apoptosis and ROS production.

\section{Conflict of Interest}

The authors declare that there is no conflict of interest to reveal.

\section{References}

Chiang YM, Chang JY, Kuo CC, Chang CY, Kuo YH. Cytotoxic triterpenes from the aerial roots of Ficus microcarpa. Phytochemistry 2005; 66: 495-501.

Daayana S, Elkord E, Winters U, Pawlita M, Roden R, Stern PL, Kitchener HC.Phase II trial of imiquimod and HPV therapeutic vaccination in patients with vulva intraepithelial neoplasia. Br J Cancer. 2010; 102: 1129-36.

Doorbar J. Molecular biology of human papillomavirus infection and cervical cancer. Clin Sci. 2006; 110: 525-41.

Huang D, Ding Y, Li Y, Zhang W, Fang W, Chen X. Antitumor activity of a 3-oxo derivative of oleanolic acid. Cancer Lett. 2006; 233: 289-96.

Kasibhatla S, Tseng B. Why target apoptosis in cancer treat- 
ment? Mol Cancer Ther. 2003; 2: 573-80.

Lowe SW, Lin AW. Apoptosis in cancer. Carcinogenesis 2000; 21: 485-95.

Ohigashi H, Takamura H, Koshimizu K, Tokuda H, Ito Y. Search for possible antitumor promoters by inhibition of 12 O-tetradecanoylphorbol-13-acetate-induced Epstein-Barr virus activation; ursolic acid and oleanolic acid from an antiinflammatory Chinese medicinal plant, Glechoma hederaceae L. Cancer Lett. 1986; 30: 143-51.

Pezzuto JM. Plant-derived anticancer agents. Biochem Pharmacol. 1997; 53: 121-33.

Prakash Chaturvedula VS, Schilling JK, Miller JS, Andriantsiferana R, Rasamison VE, Kingston DG. New cytotoxic alkaloids from the wood of Vepris punctata from the Madagascar Rainforest. J Nat Prod. 2003; 66: 532-34.

Reddy L, Odhav B, Bhoola KD. Natural products for cancer prevention: A global perspective. Pharmacol Ther. 2003; 99: 1 $-13$.
Somova LO, Nadar A, Rammanan P, Shode FO. Cardiovascular, antihyperlipidemic and antioxidant effects of oleanolic and ursolic acids in experimental hypertension. Phytomedicine 2003; 10: 115-21.

Suzuki E, Sun J, Kapoor V, Jassar As, Albelda SM. Gemcitabine has significant immunomodulatory activity in murine models independent of its cytotoxic effects. Cancer Biol Ther. 2007; 6: 880-85.

Tokuda H, Ohigashi H, Koshimizu K, Ito Y. Inhibitory effects of ursolic and oleanolic acid on skin tumor promotion by 12 O-tetradecanoylphorbol-13-acetate. Cancer Lett. 1986; 33: 279 -85 .

Wong YH, Abdul Kadir H. Induction of mitochondriamediated apoptosis in Ca Ski human cervical cancer cells triggered by mollic acid arabinoside isolated from Leea indica. Evid Based Complement Alternat Med. 2012; 2012: 684-740.

World Health Organization. Comprehensive cervical cancer control: A guide to essential practice. Geneva, WHO, 2006. 